\title{
A rationalized definition of tumor suppressor microRNAs excludes miR-34a
}

\author{
Sophie Mockly ${ }^{1}$, Élisabeth Houbron ${ }^{1}$ and Hervé Seitz ${ }^{1,2}$
}

1. Institut de Génétique Humaine, UMR 9002 CNRS and university of Montpellier, Montpellier, France

2. Corresponding author; telephone: (+33)434359936; fax: (+33)434359901;

email: herve.seitz@igh.cnrs.fr

\section{Character count for main text (including spaces): 16,505}

Running title: miR-34a is not a tumor suppressor

\section{Abstract}

While several microRNAs (miRNAs) have been proposed to act as tumor suppressors, a consensual definition of tumor suppressing miRNAs is still missing. Similarly to coding genes, we propose that tumor suppressor miRNAs must show evidence of genetic or epigenetic inactivation in cancers, and exhibit an anti-proliferative activity under endogenous expression levels. Here we observe that this definition excludes the most extensively studied tumor suppressor candidate miRNA, miR-34a. In analyzable cancer types, miR-34a does not appear to be down-regulated in primary tumors relatively to normal adjacent tissues. Deletion of miR-34a is occasionally found in human cancers, but it does not seem to be driven by an anti-tumorigenic activity of the miRNA, since it is not observed upon smaller, miR-34a-specific alterations. Its anti-proliferative action was observed upon large, supra-physiological transfection of synthetic miR-34a in cultured cells, and our data indicates that endogenous miR-34a levels do not have such an effect. Our results therefore argue against a tumor suppressive function for miR-34a, providing an explanation to the lack of efficiency of synthetic miR-34a administration against solid tumors.

Keywords: microRNA / miR-34 / proliferation / tumor suppressor 


\section{Introduction}

Tumor suppressors are genes whose activity antagonizes tumorigenesis. Consequently, they are frequently silenced, either by germline-inherited or somatic mutation, or otherwise inactivated, in cancers Green, 1988. Mechanistically, tumor suppressors mediate cellular environment-induced inhibition of cell proliferation, therefore exhibiting anti-proliferative activity under their natural expression levels: a gene displaying cytotoxic or cytostatic activity only when inappropriately overexpressed is therefore excluded from that definition [Weinberg, 1991.

miRNAs are small regulatory RNAs, guiding their effector proteins to specific target RNAs, which are repressed by various mechanisms (target RNA degradation and translational inhibition) Iwakawa and Tomari, 2015. Targets are recognized by sequence complementarity, with most targets bearing a perfect match to the miRNA "seed" (nt 2-7) Bartel, 2009. Such a short binding motif makes miRNA/target binding poorly specific, and more than $60 \%$ of human genes are predicted to be targeted by at least one miRNA Friedman et al., 2009. Because such gene regulators can act in signal transduction cascades, they may participate in tumor-suppressive pathways. A consensual definition for "tumor suppressor miRNAs" is still lacking, with some tentative definitions being based on miRNA down-regulation in cancer cells [Zhang et al., 2007], on the targets' annotation Wong et al., 2011, or both Adams et al., 2016. We rather propose to follow the initial definition of tumor suppressors Weinberg, 1991, considering that there is no reason to particularize miRNAs among other types of tumor suppressors. We thus advocate for the following definition of tumor suppressor miRNAs: (i) there is evidence for their frequent inactivation in cancer (either by genetic or epigenetic alteration; potentially only in specific cancer types); and (ii) they repress cell proliferation under their endogenous expression level, rather than upon unrealistic overexpression.

We applied this definition to interrogate the status of the most highly-studied tumor suppressor candidate miRNA, miR-34a. It is a member of the miR-34 family, comprising six members in human and in mouse: miR-34a, miR-34b, miR-34c, miR-449a, miR-449b and miR-449c (Supplementary Figure S1). The three miR-34a/b/c subfamily members are transcriptionally controlled by the p53 tumor suppressor, which suggested that these miRNAs could participate in the tumor suppressive activity of the p53 network He et al., 2007, Bommer et al., 2007, Chang et al., 2007, Corney et al., 2007, Tarasov et al., 2007, Tazawa et al., 2007, Raver-Shapira et al., 2007. Indeed, the miR-34a member is down-regulated or lost in various cancer models (tumor samples or transformed cell lines) relatively to normal samples [He et al., 2007, Chang et al., 2007, Welch et al., 2007, Bommer et al., 2007, Tazawa et al., 2007, Lodygin et al., 2008, Gallardo et al., 2009, Wiggins et al., 2010, Corney et al., 2010. Yet $m i R-34 a^{-1-}, m i R-34 b^{-/-}, m i R-34 c^{-/-}$triple knock-out mice do not exhibit obvious defects in p53-dependent proliferation control or in tumor suppression Concepcion et al., 2012. And, while pre-clinical studies in mice gave encouraging results (reviewed in [Bader, 2012, Hong et al., 2020]), administration of a synthetic miR-34a to human patients with solid tumors failed to repress tumor growth reproducibly Beg et al., 2017. An alternate administration regimen (allowing increased drug exposure) did not clearly improve clinical outcomes, while triggering poorly-understood, severe adverse effects [Hong et al., 2020].

\section{Results and Discussion}

It is now possible to compare miRNA levels between tumors and normal adjacent tissues on a large collection of human cases Zhang et al., 2021, allowing a rigorous assessment of miR-34a expression in tumorigenesis. Selecting every cancer type where miRNA expression is available for primary tumor and normal adjacent tissue, in at least 10 studied cases ( $\mathrm{n}=20$ cancer types), we did not find any cancer type where miR-34a was significantly down-regulated (Fig $1 A$ ). Hence in this collection of cancer types, human primary tumors do not tend to under-express miR-34a, contradicting the notion that genetic or epigenetic silencing of miR-34a could participate in tumorigenesis.

Accordingly, genetic alterations affecting $m i R-34 a$ are very rare in cancer: focusing on every cancer type for which gene-level copy number was measured in at least 100 cases $(n=29$ cancer types), we did not observe any tendency for the loss of miR-34a relatively to other miRNA genes 
bioRxiv preprint doi: https://doi.org/10.1101/2021.02.11.430795; this version posted June 15, 2021. The copyright holder for this preprint (which was not certified by peer review) is the author/funder, who has granted bioRxiv a license to display the preprint in perpetuity. It is made available under aCC-BY-ND 4.0 International license.
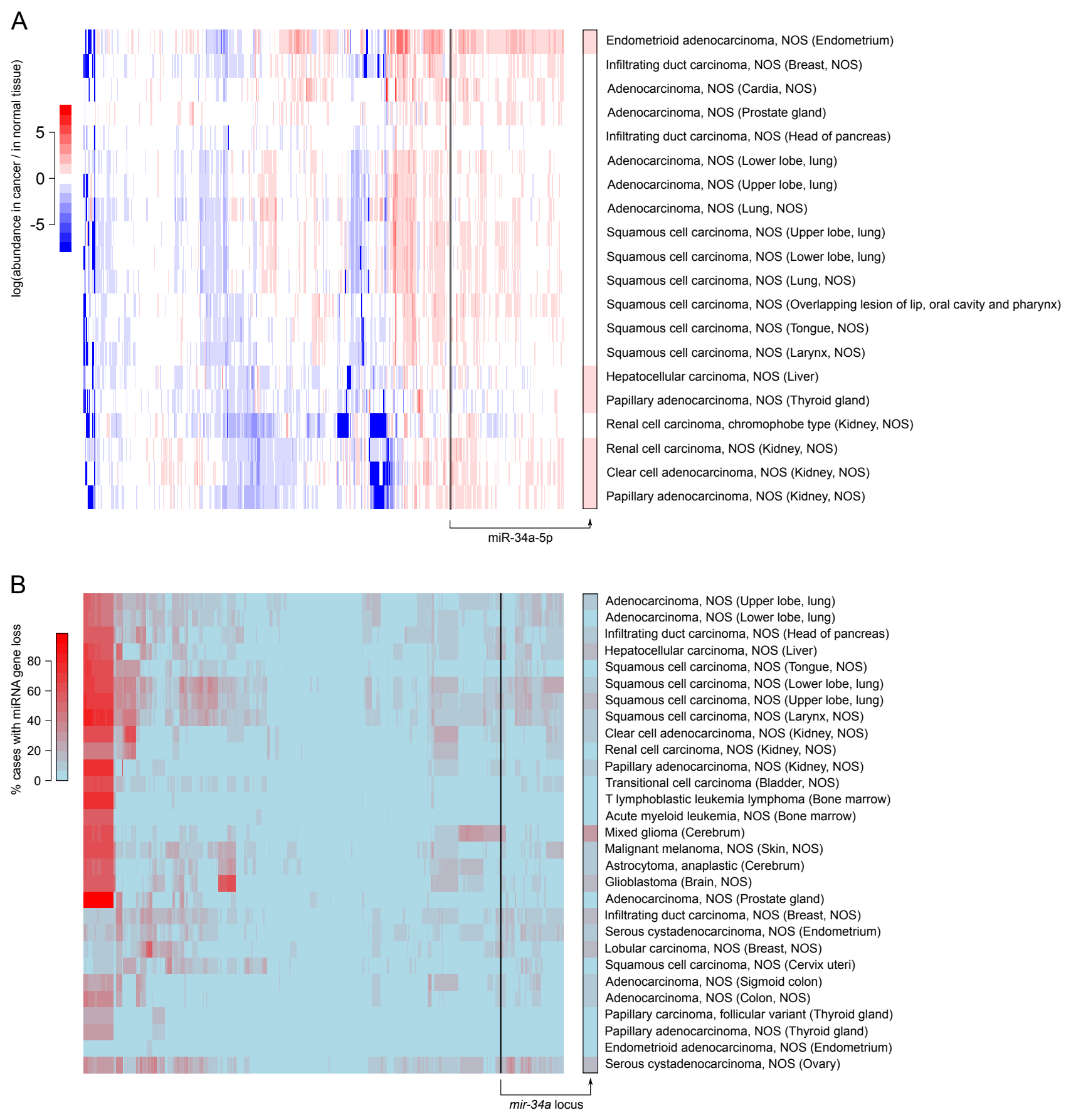

Figure 1: mir-34a is not generally down-regulated or lost in cancers. (A) miRNA abundance (normalized by the number of mapped miRNA reads) was compared between primary tumors and normal adjacent tissues. Only cancer types for which at least 10 cases were analyzed have been considered ( $n=20$ cancer types; rows), and miRNAs with a null variance across cancer types were excluded (remaining: $n=545$ miRNAs; columns). For each miRNA/cancer type pair, the heatmap shows its median fold-change across all cases, with non-significant changes (FDR $\geqslant 0.05)$ being shown in white. $\log$ (fold-changes) larger than +8 or smaller than -8 were set to +8 or -8 respectively, for graphical clarity. (B) Only cancer types for which at least 100 cases were analyzed have been considered $(\mathrm{n}=29$ cancer types; rows), and miRNA genes whose ploidy could not be assessed were excluded (remaining: $\mathrm{n}=1,686$ miRNAs; columns). For each miRNA/cancer type pair, the heatmap shows the percentage of cases with monoallelic or biallelic loss of the miRNA gene. Both panels: the column showing miR-34a data is magnified on the right margin (framed in black). "NOS": not otherwise specified. 
(see Fig $1 B$ ). Similarly, we did not find any evidence for the selective mutation of the pre-miR-34a hairpin precursor sequence, mature miR-34a or the miR-34a seed in cancers $(\mathrm{n}=30$ analyzed cancer types; Supplementary Figure S2). In contrast to miR-34a, 105 miRNA loci tend to be frequently lost in 19 cancer types (red area at the top left corner of the heatmap in Fig $1 B$; listed in Supplementary Table S1): these miRNAs are more convincing tumor suppressor candidates than miR-34a in this respect.

Hence the loss or mutation of miR-34a does not appear to be enriched in cancer. We note that $m i R-34 a$ is located on cytogenetic band $1 \mathrm{p} 36$, which is often altered in a wide variety of cancers. But our analyses suggest that the inactivation of $m i R-34 a$ is not the actual driver for deletion selection - and because a convincing tumor suppressor is already known at 1p36 (the CHD5 gene [Bagchi et al., 2007]), we propose that the occasional deletion of miR-34a in cancer is rather a consequence of its genomic proximity with such a real tumor suppressor. Accordingly, whenever a limited region of consistent deletion could be mapped in $1 \mathrm{p} 36$, that region excludes miR-34a (with the only exception of myelodysplastic syndromes, but with low experimental support): see Supplementary Figure S3.

miR-34a has also been considered a tumor suppressor candidate on the basis of the apparent anti-proliferative activity of miR-34 family miRNAs. Numerous studies in cultured cell lines indeed showed that miR-34 transfection inhibits cell proliferation WWelch et al., 2007, He et al., 2007, Chang et al., 2007, Corney et al., 2007, Tarasov et al., 2007, Tazawa et al., 2007, Raver-Shapira et al., 2007.

But miRNA over-expression generates false positives, raising the possibility that this reported antiproliferative role is artifactual Mockly and Seitz, 2019]. We thus deleted the miR-34a gene in HCT-116 cells, where it has been proposed to be anti-proliferative by several independent studies He et al., 2007, Chang et al., 2007, Tazawa et al., 2007 (mutagenesis strategy in Supplementary Figure S4). Deletion of the miR-34a locus eliminated $94 \%$ of the expression of the whole miR-34 family (Fig $2 A$ and $B$ ). Our results do not show any significant difference in the growth rate of $m i R$ $34 a^{-/-}$and wild-type clones $\left(\right.$Fig $2 C$ ). We also prepared $m i R-34 a^{-}$clones from the human haploid HAP1 cell line, where miR-34a is also not anti-proliferative (it is even slightly pro-proliferative; Supplementary Figure S5). It could be argued that miR-34a does not inhibit cell proliferation in unstressed conditions, while being anti-proliferative upon genotoxic stress. But we also failed to observe significant differences between wild-type and mutant clones under doxorubicin or 5-fluoro-uracil treatment (Fig $2 D$ and $E$ ).

In agreement with published data, we did observe a strong reduction in cell proliferation when we transfected HCT-116 cells with large amounts (10 nM) synthetic miR-34a duplex (Fig 3A), but that effect was lost when transfecting $1 \mathrm{nM}$ duplex (Fig $3 B$ ). Absolute miRNA quantification by RTddPCR shows that a $10 \mathrm{nM}$ transfection over-expresses miR-34a by $>8,000$-fold in HCT-116 cells (and a $1 \mathrm{nM}$ transfection over-expresses it by $>490$-fold), clearly demonstrating that such an experiment results in supra-physiological miRNA concentrations (Fig $3 C$ ). For comparison, we measured the increase in miR-34a expression in response to DNA damage: a $72 \mathrm{~h}$ treatment with doxorubicin at its IC50 concentration $\left(7 \times 10^{-8} \mathrm{M}\right.$ in HCT-116 cells; Supplementary Figure S6) over-expresses miR-34a by only 4.7 -fold (Fig $3 D)$.

Of note, some authors have previously characterized the proliferative effect of miR-34 using genetic ablation rather than over-expression. In one study, mouse embryonic fibroblasts (MEFs) devoid of miR-34a/b/c appear to grow at the same rate than wild-type MEFs, except, transiently, for one early time-point Concepcion et al., 2012. In another study, genetic inactivation of the miR$34 a$ gene in HCT-116 is reported to accelerate cell proliferation, in stark contrast with our own findings Navarro and Lieberman, 2015. Such discrepancy would deserve to be investigated, but unfortunately that published mutant cell line has been lost and it is no longer available from the authors (Dr. J. Lieberman, personal communication).

While the miR-34 family is believed to exert a tumor suppressive action in a diversity of cancers Slack and Chinnaiyan, 2019, we observed that it is hardly expressed in cultured cell lines, primary tissues and body fluids (Supplementary Figure S7-S9). Current RNA detection technologies can be extremely sensitive, and they can detect miRNAs which are too poorly abundant to induce any clear 
bioRxiv preprint doi: https://doi.org/10.1101/2021.02.11.430795; this version posted June 15, 2021. The copyright holder for this preprint (which was not certified by peer review) is the author/funder, who has granted bioRxiv a license to display the preprint in perpetuity. It is made available under aCC-BY-ND 4.0 International license.
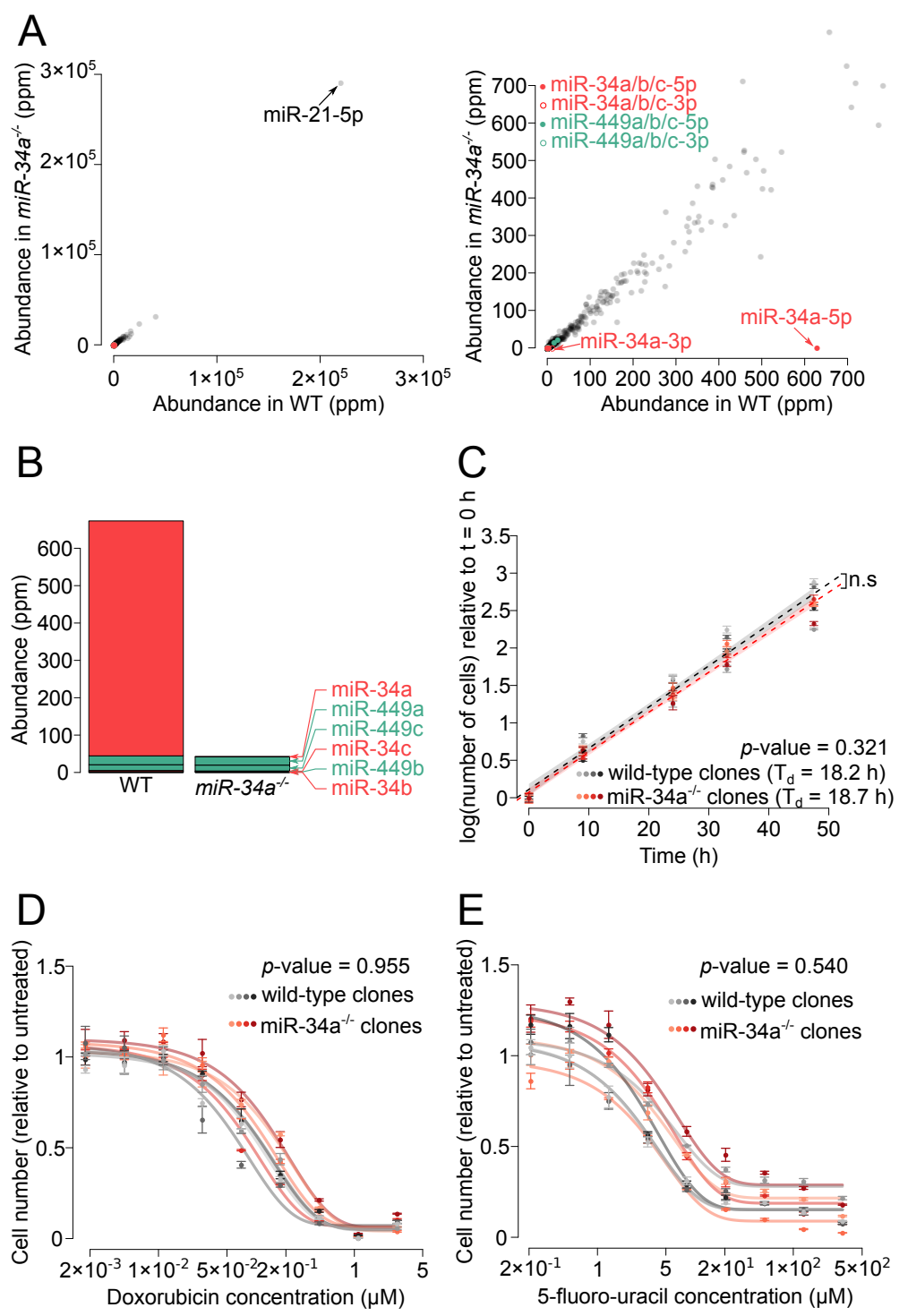

Figure 2: miR-34 is not a general repressor of cell proliferation. (A) miRNA quantification by Small RNA-Seq in a representative wild-type HCT-116 clone $\left(x\right.$ axis) and a representative $m i R$ - $34 a^{-/-}$ clone ( $y$ axis). Right panel: magnification of the left panel. (B) Cumulated abundance of miR-34 family members in the two clones. miRNAs are sorted vertically according to their abundance in the wild-type clone. (C) Four wild-type and four miR-34a mutant clones were grown in sub-confluent conditions. Means and standard errors of 4 biological replicates are represented by dots and error bars. Linear modeling of log-transformed cell counts relative to time was used to measure doubling time $\left(\mathrm{T}_{d}\right)$, and to estimate the significance of the effect of genotype ( $p$-value is given in the inset). Shaded areas represent the $95 \%$ confidence interval for theoretical future measurements. $(D, E)$ Cell number after 3 days of culture in presence of varying doses of $(D)$ doxorubicin or $(E)$-fluoro-uracil (4 clones of each genotype were analyzed; 3 biological replicates for each drug concentration; mean $+/$ - st. error is shown). Cell number was normalized to cell number count in untreated replicates. Normalized cell number was fitted to an asymptotic model for each clone (fitted models are represented by curves). In order to assess the significance of the effect of genotype, a naïve (non-informed by clone genotype) and a genotype-informed model were compared by an analysis of variance ( $p$-value is indicated in the inset).

change in target expression Mullokandov et al., 2012. Hence we anticipate that in all the cell lines for which we analyzed miRNA abundance, and in most cells in the analyzed tissues, miR-34 family miRNAs are actually non-functional. 
Yet we do not question the overall functionality of miR-34 miRNAs in vivo. Because that family is deeply conserved in evolution (shared between, e.g., vertebrates and insects), it certainly plays important biological functions, perhaps only in a small number of cells, or at very specific developmental stages, where its abundance would be high enough. In mouse, the miR-34 family is particularly expressed in lungs and testes Concepcion et al., 2012, Song et al., 2014. Mutation of all 6 members of the miR-34 family causes severe ciliogenesis defects, leading to respiratory distress and impaired gametogenesis - translating into sterility and premature mortality [Song et al., 2014]. Unsurprisingly then, the most obvious biological functions of that miRNA family seem to take place in the tissues where miR-34 miRNAs are highly expressed, in contrast with the widely-accepted notion of their broad anti-tumorigenic activity.

While the original definition for tumor suppressors had been formulated with coding genes in mind, we consider that there is no objective reason for adopting a different definition for tumor suppressor miRNAs. In this view, the most heavily studied candidate tumor suppressor miRNA, miR-34a, does not appear to be a tumor suppressor. It remains formally possible that miR-34a inactivation is frequent in specific cancer types, distinct from those we could analyze in Fig 1 and Supplementary Figure S2. In that case, miR-34a may be a tumor suppressor in these particular cancers - but this possibility is merely speculative, and no such cancer type has been identified so far.

We confirmed that a large artificial over-expression $(10 \mathrm{nM})$ of miR-34a indeed represses cell proliferation. It could be argued that this cytotoxic effect could provide the ground for an efficient anti-cancer treatment, no matter how un-natural it is. But the whole purpose of using natural tumor suppressors (e.g., miRNAs) is that they are expected to be well tolerated, because they already exist endogenously. Administering large amounts of cytotoxic agents to patients may indeed kill cancer cells - but it will also likely trigger unwanted adverse effects. In this view, synthetic miR34a behaves similarly to existing anti-cancer drugs, which are based on exogenous molecules. It is therefore not surprising to observe a variety of adverse secondary effects when the MRX34 miR-34a mimic is administered to patients Beg et al., 2017, Hong et al., 2020. More inocuous miRNA-based treatments may be possible, but they would have to rely on rigorously established tumor-suppressive activity of the endogenous miRNA.

\section{Materials and Methods}

\section{Analysis of $m i R-34 a$ expression and integrity in human cancers}

miRNA expression data was downloaded from the GDC portal on April 29, 2021. Cancer types where at least 10 cases were available (with Small RNA-Seq data from normal solid tissue and primary tumor for each case) were selected, and depth-normalized read counts were compared between normal tissue and tumor for each case. The heatmap shown on Fig $14 A$ shows the median log-ratio between tumor and normal tissue, with non-significant changes (calculated with the Wilcoxon test, FDR-adjusted for multiple hypothesis testing) being colored in white.

miRNA gene ploidy data was downloaded from the GDC portal on March 4, 2021. Erroneous miRNA gene coordinates were corrected using information from miRBase. For the heatmap shown on Fig $1 B$, the percentage of cases with miRNA gene loss (either homo- or heterozygous) was evaluated for each miRNA, selecting cancer types where ploidy was determined in at least 100 cases.

miRNA sequence variation data was downloaded from the GDC portal on February 24, 2021. SNP location was intersected with miRNA hairpin and mature miRNA coordinates from miRBase (as well as with miRNA seed coordinates, defined as nt 2-7 of the mature miRNA). For the heatmaps shown on Supplementary Figure S2, the percentage of cases with sequence variations in miRNA genes (hairpin, mature or seed sequences) is displayed, selecting cancer types with at least 100 analyzed cases.

For each of these heatmaps, miRNAs and cancer types were clustered with the heatmap. 2 command with the $\mathbf{R}$ software. 


\section{CRISPR/Cas9-mediated mutagenesis}

Four sgRNAs were designed using CRISPOR (http://crispor.tefor .net/ Concordet and Haeussler, 2018) to target each side of the human pre-mir-34a sequence, and cloned into an expression plasmid for S. pyogenes Cas9 (pSpCas9(BB)-2A-GFP plasmid (PX458), a gift from Feng Zhang [Ran et al., 2013; Addgene plasmid \#48138; http://n2t.net/addgene:48138; RRID:Addgene_48138). Targeting efficiency of each plasmid was estimated by Sanger sequencing of the targeted locus in transfected HCT-116 cells, and analyzed with the Synthego ICE Analysis online tool (https://ice.synthego. $\mathrm{com} / \# /$ ). Mutagenesis was performed using the most efficient sgRNA sequence on each side of the targeted locus (AAGCTCTTCTGCGCCACGGTGGG and GCCGGTCCACGGCATCCGGAGGG; PAM sequences in bold; also see Supplementary Figure S4).

HCT-116 (ATCC® cat. \#CCL247) and HAP1 (Horizon Discovery cat. \#C631) cells were grown till $80 \%$ confluency and transfected with the two plasmids (15 $\mathrm{\mu g}$ each) following the protocol for Lipofectamine 2000 Transfection Reagent (Thermo Fisher Scientific). After 24 hours, Cas9-GFPexpressing single cells were isolated in 96-well plates by flow cytometry on a BD FACSMelody (Becton Dickinson), then grown for 10 days. Homozygous wild-type and mutant clones were first tested by discriminative PCRs (with primer pairs ACTTCTAGGGCAGTATACTTGCT and GCTGTGAGTGTTTCTTTGGC; and TCCTCCCCACATTTCCTTCT and GCAAACTTCTCCCAGCCAAA), and eventually validated by Sanger sequencing of their miR-34a locus. For the HAP1 cell line, mutagenesis efficiency was so high that we were unable to isolate wild-type clones after cotransfection of sgRNA-carrying PX458 plasmids. Wild-type clones were therefore generated by transfection of HAP1 cells with a plasmid expressing SpCas9-HF1 variant but no sgRNA (the VP12 plasmid, a gift from Keith Joung Kleinstiver et al., 2016]; Addgene plasmid \#72247 ; http://n2t.net/addgene: 72247; RRID:Addgene_72247), and went through the same isolation and selection process as mutant clones.

\section{RNA extraction}

Cells plated in $10 \mathrm{~cm}$ Petri dishes were lysed and scrapped in $6 \mathrm{~mL}$ ice-cold TRIzol ${ }^{\mathrm{TM}}$ Reagent (Invitrogen) added directly to the culture dish after removal of the growth medium, and mixed with $1.2 \mathrm{~mL}$ of water-saturated chloroform. Samples were homogenized by vigorous shaking for $1 \mathrm{~min}$ and centrifuged for $5 \mathrm{~min}$ at $12,000 \mathrm{~g}$ and $4^{\circ} \mathrm{C}$ to allow phase separation. The aqueous phase was transfered in a new tube and mixed with $3 \mathrm{~mL}$ isopropanol for precipitation. After a 10 min incubation at room temperature, samples were centrifuged for $10 \mathrm{~min}$ at $12,000 \mathrm{~g}$ and $4{ }^{\circ} \mathrm{C}$ and the supernatant was removed. The RNA pellet was washed with $6 \mathrm{~mL}$ of $70 \%$ ethanol and samples were centrifuged for 5 min at $12,000 \mathrm{~g}$ and $4^{\circ} \mathrm{C}$. After complete removal of ethanol, the RNA pellet was resuspended in $20 \mu \mathrm{L}$ RNase-free water and the quantity of total RNA was determined by spectrophotometry on a NanoDrop ND-1000.

\section{Small RNA-Seq}

Total RNA of each cell line was extracted $48 \mathrm{~h}$ after seeding and quality was assessed on electrophoretic spectra from a Fragment Analyzer (Agilent), analyzed with the PROSize software (v. 3.0.1.6). Libraries were prepared using NEXTflex ${ }^{\mathrm{TM}}$ Small RNA-Seq Kit v3 (Bioo Scientific) following the manufacturer's instructions. Libraries were verified by DNA quantification using Fragment Analyzer (kit High Sensitivity NGS), and by qPCR (ROCHE Light Cycler 480). Libraries were sequenced on Illumina NovaSeq 6000 using NovaSeq Reagent Kit (100 cycles). RNA quality assessment, library preparation, validation and sequencing were performed by the MGX sequencing facility.

Adapters ended with 4 randomized nucleotides in order to reduce ligation biases. Because of the sequencing design, the adapter sequence (5' GTTCAGAGTTCTACAGTCCGACGATCNNNN 3’) appears at the beginning of the read sequence, and the final 4 nucleotides of the read are the initial randomized nucleotides of the other adapter, whose other nucleotides are not read. Hence small RNA reads can be extracted from the fastq files with the following command: 
bioRxiv preprint doi: https://doi.org/10.1101/2021.02.11.430795; this version posted June 15,2021 . The copyright holder for this preprint (which was not certified by peer review) is the author/funder, who has granted bioRxiv a license to display the preprint in perpetuity. It is made available under aCC-BY-ND 4.0 International license.

cutadapt -g GTTCAGAGTTCTACAGTCCGACGATCNNNN --discard-untrimmed -m 18 -M 30 । \$input_file.fastq | cutadapt $-\mathrm{u}-4-$

\section{Cell transfection}

Cells were transfected 24 hours after seeding either with a control duplex, siRNA against eGFP: 5'-GGCAAGCUGACCCUGAAGUdTdT-3' / 5'-ACUUCAGGGUCAGCUUGCCdTdT-3' or with a hsa-miR-34a mimic duplex: 5'-P-UGGCAGUGUCUUAGCUGGUUGUU-3' / 5'-P-CAAUCAGCAAGUAUACUGCCCUA-3' according to the protocol for Lipofectamine 2000 Transfection Reagent (Thermo Fisher Scientific).

\section{Proliferation assays}

Because the mere procedure of isolating and selecting mutated clones may artifactually select clones with exceptionally high proliferation rates, we applied the same isolation and selection procedure to wild-type clones, and we measured proliferation rates on several independent wild-type and mutant clones. Each cell line was seeded in 96-well plates (Fig 26 : in 4 replicates at $3 \times 10^{3}$ cells/well per time point; Figs $3 A$ and $B$ : in 6 replicates at $6 \times 10^{3}$ cells/well). From 24 hours after cell seeding or transfection, to 3 days later, the number of living cells was determined twice a day by CellTiter-Glo Luminescent Cell Viability Assay (Promega) according to the manufacturer's protocol and recorded with a TriStar LB 941 (Berthold Technologies). Linear regression of log-transformed cell counts relative to time and genotype (in $\mathbf{R}$ syntax: log-transformed cell counts time $*$ genotype) or transfected duplex identity (log-transformed cell counts time $*$ duplex identity) was used to measure doubling time and to estimate the significance of the effect of genotype or transfected duplex.

For Fig $2 D$ and $E$, doxorubicin (Sigma-Aldrich) was diluted in molecular biology-grade water and 5-fluorouracil (5-FU) (Sigma-Aldrich) diluted in dimethyl sulfoxide (SigmaAldrich). In a preliminary experiment, half-maximal inhibitory concentration (IC50) was estimated after $72 \mathrm{~h}$ drug exposure: $7 \times 10^{-8} \mathrm{M}$ and $8 \times 10^{-6} \mathrm{M}$ for doxorubicin and 5 -FU respectively. Cell lines were seeded in 3 replicates per drug concentration at $2.5 \times 10^{3}$ cells/well in 96-well plates. After 24 hours, culture medium was replaced with drug-containing medium (concentration range centered on the IC50 with $2.5 \times$ increments), or solvant-containing medium for untreated controls, and the number of living cells was determined $72 \mathrm{~h}$ later by CellTiterGlo Luminescent Cell Viability Assay (Promega). Cell counts were normalized to the mean cell number in untreated controls. Normalized cell number was fitted to an asymptotic model for each clone to assess the significance of the effect of genotype (using an analysis of variance to compare a model not informed by clone genotype, to a genotype-informed model).

\section{miRNA quantification by RT-ddPCR}

Reverse transcription of a specific miRNA in HCT-116 cells was performed on 10 ng total RNA using the TaqMan microRNA Reverse Transcription Kit (Thermo Fisher Scientific) in a total volume of $15 \mu \mathrm{L}$, according to the manufacturer's protocol, with miRNA-specific RT primers from the TaqMan MicroRNA Assay Kit (assay IDs for hsa-miR-34a-5p and miR-21b-5p are respectively 000426 and 000397). ddPCR amplification of the cDNA was performed on $1.33 \mu \mathrm{L}$ of each cDNA combined with $1 \mu \mathrm{L}$ of miRNA-specific 20X TaqMan MicroRNA Reagent containing probes and primers for amplification from the TaqMan MicroRNA Assay Kit (Thermo Fisher Scientific), $10 \mu \mathrm{L}$ of $2 \mathrm{X}$ ddPCR Supermix for probes (no dUTP) (Bio-Rad), and $7.67 \mu \mathrm{L}$ of molecular biology-grade water. Droplets were generated, thermal cycled and detected by the QX200 Droplet Digital PCR System (Bio-Rad) according to the ddPCR Supermix protocol and manufacturer's instructions. Data were extracted using QuantaSoft Pro Software (Bio-Rad). 


\section{Data and script availability}

Deep-sequencing data has been deposited at SRA and linked to BioProject number PRJNA695193. Scripts, raw, intermediate and final data files are available at https://github.com/HKeyHKey/ Mockly_et_al_2021 and at https://www.igh.cnrs.fr/en/research/departments/genetics-development/ systemic-impact-of-small-regulatory-rnas\#programmes-informatiques/

\section{Acknowledgments}

We thank G. Canal, C. Theillet, P.D. Zamore and members of the Seitz lab for critical reading of the manuscript, and A. Pélisson and K. Mochizuki for useful discussions. This research was supported by Cancéropôle GSO "Émergence" grant and Projet Fondation ARC \#PJA 20191209613. We thank the MGX facility (Biocampus Montpellier, CNRS, INSERM, Univ. Montpellier, Montpellier, France) for sequencing the Small RNA-Seq libraries.

\section{Author contributions}

S.M. and É.H. performed experiments; S.M. and H.S. performed computational analyses; S.M. and H.S. wrote the manuscript and prepared figures.

\section{Conflict of interest}

The authors do not declare any conflict of interest.

\section{References}

[Adams et al., 2016] Adams, B. D., Parsons, C. and Slack, F. J. (2016). Expert Opin Ther Targets 20, 737-753.

[Bader, 2012] Bader, A. G. (2012). Front Genet 3, 120.

[Bagchi et al., 2007] Bagchi, A., Papazoglu, C., Wu, Y., Capurso, D., Brodt, M., Francis, D., Bredel, M., Vogel, H. and Mills, A. A. (2007). Cell 128, 459-475.

[Bartel, 2009] Bartel, D. P. (2009). Cell 136, 215-233.

[Beg et al., 2017] Beg, M. S., Brenner, A. J., Sachdev, J., Borad, M., Kang, Y.-K., Stoudemire, J., Smith, S., Bader, A. G., Kim, S. and Hong, D. S. (2017). Invest New Drugs 35, 180-188.

[Bommer et al., 2007] Bommer, G. T., Gerin, I., Feng, Y., Kaczorowski, A. J., Kuick, R., Love, R. E., Zhai, Y., Giordano, T. J., Qin, Z. S., Moore, B. B., MacDougald, O. A., Cho, K. R. and Fearon, E. R. (2007). Curr Biol 17, 1298-1307.

[Chang et al., 2007] Chang, T.-C., Wentzel, E. A., Kent, O. A., Ramachandran, K., Mullendore, M., Lee, K. H., Feldmann, G., Yamakuchi, M., Ferlito, M., Lowenstein, C. J., Arking, D. E., Beer, M. A., Maitra, A. and Mendell, J. T. (2007). Mol Cell 26, 745-752.

[Concepcion et al., 2012] Concepcion, C. P., Han, Y.-C., Mu, P., Bonetti, C., Yao, E., D'Andrea, A., Vidigal, J. A., Maughan, W. P., Ogrodowski, P. and Ventura, A. (2012). PLoS Genet 8, e1002797.

[Concordet and Haeussler, 2018] Concordet, J.-P. and Haeussler, M. (2018). Nucleic Acids Res 46, W242-W245.

[Corney et al., 2007] Corney, D. C., Flesken-Nikitin, A., Godwin, A. K., Wang, W. and Nikitin, A. Y. (2007). Cancer Res 67, 8433-8438. 
[Corney et al., 2010] Corney, D. C., Hwang, C.-I., Matoso, A., Vogt, M., Flesken-Nikitin, A., Godwin, A. K., Kamat, A. A., Sood, A. K., Ellenson, L. H., Hermeking, H. and Nikitin, A. Y. (2010). Clin Cancer Res 16, 1119-1128.

[Friedman et al., 2009] Friedman, R. C., Farh, K. K., Burge, C. B. and Bartel, D. P. (2009). Genome Res 19, 92-105.

[Gallardo et al., 2009] Gallardo, E., Navarro, A., Viñolas, N., Marrades, R. M., Diaz, T., Gel, B., Quera, A., Bandres, E., Garcia-Foncillas, J., Ramirez, J. and Monzo, M. (2009). Carcinogenesis 30, 1903-1909.

[Green, 1988] Green, A. R. (1988). Br J Cancer 58, 115-121.

[He et al., 2007] He, L., He, X., Lim, L. P., de Stanchina, E., Xuan, Z., Liang, Y., Xue, W., Zender, L., Magnus, J., Ridzon, D., Jackson, A. L., Linsley, P. S., Chen, C., Lowe, S. W., Cleary, M. A. and Hannon, G. J. (2007). Nature 447, 1130-1134.

[Hong et al., 2020] Hong, D. S., Kang, Y.-K., Borad, M., Sachdev, J., Ejadi, S., Lim, H. Y., Brenner, A. J., Park, K., Lee, J.-L., Kim, T.-Y., Shin, S., Becerra, C. R., Falchook, G., Stoudemire, J., Martin, D., Kelnar, K., Peltier, H., Bonato, V., Bader, A. G., Smith, S., Kim, S., O'Neill, V. and Beg, M. S. (2020). Br J Cancer 122, 1630-1637.

[Iwakawa and Tomari, 2015] Iwakawa, H. O. and Tomari, Y. (2015). Trends Cell Biol 25, 651-665.

[Kleinstiver et al., 2016] Kleinstiver, B. P., Pattanayak, V., Prew, M. S., Tsai, S. Q., Nguyen, N. T., Zheng, Z. and Joung, J. K. (2016). Nature 529, 490-495.

[Lodygin et al., 2008] Lodygin, D., Tarasov, V., Epanchintsev, A., Berking, C., Knyazeva, T., Körner, H., Knyazev, P., Diebold, J. and Hermeking, H. (2008). Cell Cycle 7, 2591-2600.

[Mockly and Seitz, 2019] Mockly, S. and Seitz, H. (2019). Methods Mol Biol 1970, 291-314.

[Mullokandov et al., 2012] Mullokandov, G., Baccarini, A., Ruzo, A., Jayaprakash, A. D., Tung, N., Israelow, B., Evans, M. J., Sachidanandam, R. and Brown, B. D. (2012). Nat Methods 9, 840-846.

[Navarro and Lieberman, 2015] Navarro, F. and Lieberman, J. (2015). PLoS One 10, e0132767.

[Ran et al., 2013] Ran, F. A., Hsu, P. D., Wright, J., Agarwala, V., Scott, D. A. and Zhang, F. (2013). Nat Protoc 8, 2281-2308.

[Raver-Shapira et al., 2007] Raver-Shapira, N., Marciano, E., Meiri, E., Spector, Y., Rosenfeld, N., Moskovits, N., Bentwich, Z. and Oren, M. (2007). Mol Cell 26, 731-743.

[Slack and Chinnaiyan, 2019] Slack, F. J. and Chinnaiyan, A. M. (2019). Cell 179, 1033-1055.

[Song et al., 2014] Song, R., Walentek, P., Sponer, N., Klimke, A., Lee, J. S., Dixon, G., Harland, R., Wan, Y., Lishko, P., Lize, M., Kessel, M. and He, L. (2014). Nature 510, 115-120.

[Tarasov et al., 2007] Tarasov, V., Jung, P., Verdoodt, B., Lodygin, D., Epanchintsev, A., Menssen, A., Meister, G. and Hermeking, H. (2007). Cell Cycle 6, 1586-1593.

[Tazawa et al., 2007] Tazawa, H., Tsuchiya, N., Izumiya, M. and Nakagama, H. (2007). Proc Natl Acad Sci USA 104, 15472-15477.

[Weinberg, 1991] Weinberg, R. A. (1991). Science 254, 1138-1146.

[Welch et al., 2007] Welch, C., Chen, Y. and Stallings, R. L. (2007). Oncogene 26, 5017-5022.

[Wiggins et al., 2010] Wiggins, J. F., Ruffino, L., Kelnar, K., Omotola, M., Patrawala, L., Brown, D. and Bader, A. G. (2010). Cancer Res 70, 5923-5930. 
bioRxiv preprint doi: https://doi.org/10.1101/2021.02.11.430795; this version posted June 15, 2021. The copyright holder for this preprint (which was not certified by peer review) is the author/funder, who has granted bioRxiv a license to display the preprint in perpetuity. It is made available under aCC-BY-ND 4.0 International license.

[Wong et al., 2011] Wong, K. Y., Yu, L. and Chim, C. S. (2011). Epigenomics 3, 83-92.

341

[Zhang et al., 2007] Zhang, B., Pan, X., Cobb, G. P. and Anderson, T. A. (2007). Dev Biol 302, $342 \quad 1-12$.

343 [Zhang et al., 2021] Zhang, Z., Hernandez, K., Savage, J., Li, S., Miller, D., Agrawal, S., Ortuno, F., 344 Staudt, L. M., Heath, A. and Grossman, R. L. (2021). Nat Commun 12, 1226. 

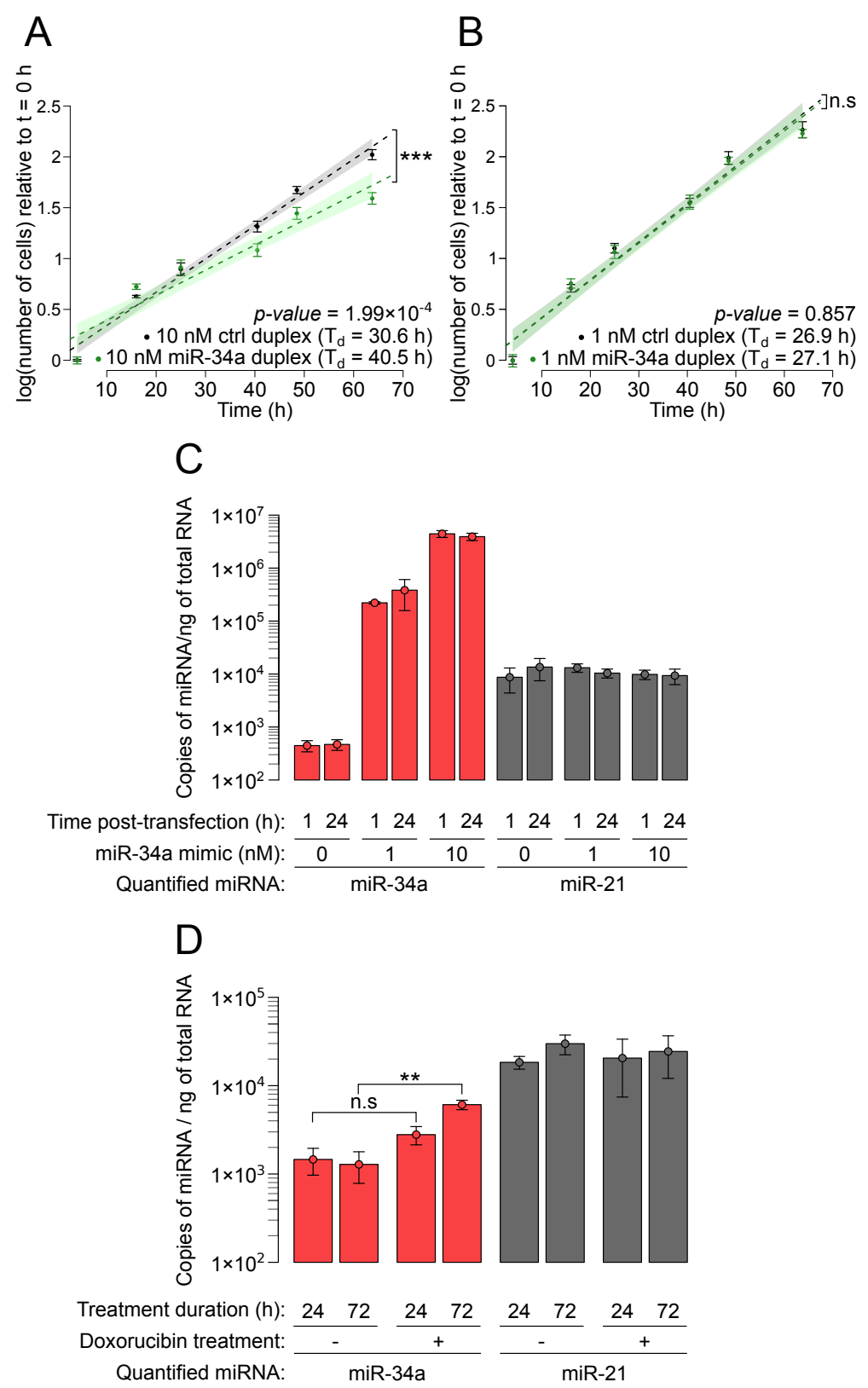

Figure 3: Supra-physiological transfection of miR-34a inhibits cell proliferation. Wild-type HCT-116 cells were transfected with $10 \mathrm{nM}$ (panel $A$ ) or $1 \mathrm{nM}$ (panel $B$ ) duplex (either a control siRNA duplex, or miR-34a/miR-34a* duplex) and grown in sub-confluent conditions. Means and standard errors of 6 biological replicates are represented by dots and error bars. Linear modeling of log-transformed cell counts relative to time was used to measure doubling time $\left(\mathrm{T}_{d}\right)$, and to estimate the significance of the effect of duplex identity ( $p$-values are given in the inset; asterisks denote $p$-value $<0.05$, "n.s." indicates larger $p$-values). Shaded areas represent the $95 \%$ confidence interval for theoretical future measurements. (C) Cellular abundance of miR-34a (red bars) or a control miRNA (miR-21; gray bars) 1 or $24 \mathrm{~h}$ after transfection of HCT-116 cells with 0,1 or $10 \mathrm{nM} \mathrm{miR-34a/miR-34a*}$ duplex. (D) HCT-116 cells were treated for 24 or $72 \mathrm{~h}$ with $7 \times 10^{-8} \mathrm{M}$ doxorubicin, and their intracellular miR-34a and miR-21 were quantified by RT-ddPCR. Two-way ANOVA analysis shows that doxorubicin treatment has an effect on miR-34a levels $(p=0.0013)$, and post-hoc pairwise t-tests find the effect significant only after $72 \mathrm{~h}$ exposure to the drug ( $p=0.0521$ for $24 \mathrm{~h}$ exposure, $p=0.00138$ for $72 \mathrm{~h}$ exposure, indicated by "n.s." and "**" respectively). A similar two-way ANOVA analysis does not detect a significant effect of doxorubicin treatment on miR-21 levels $(p=0.768)$. (Panels $C$ and $D$ ) Means and standard errors of 3 biological replicates are represented by dots and error bars, respectively. 